\title{
Expression of CK7 and CDKN2 in Cervical Intraepithelial Neoplasia and Correlation with Clinical Outcome
}

\author{
TATIANA MEGALE DE LIMA ${ }^{1}$, GUSTAVO RUBINO DE AZEVEDO FOCCHI ${ }^{2}$, BRUNA CRISTINE DE ALMEIDA ${ }^{3}$, \\ PATRICIA NAPOLI BELFORT-MATTOS ${ }^{1}$, THAIS HEINKE ${ }^{2}$ and NEILA MARIA DE GÓIS SPECK ${ }^{1}$ \\ ${ }^{1}$ Gynecological Disease Prevention Nucleus (NUPREV), Department of Gynecology, \\ Federal University of São Paulo, Sao Paulo, Brazil; \\ ${ }^{2}$ Department of Pathology, Federal University of São Paulo, Sao Paulo, Brazil; \\ ${ }^{3}$ Structural and Molecular Gynecology Laboratory (LIM 58), Department of Obstetrics and Gynecology, \\ Clinical Hospital of University of São Paulo Medical School, Sao Paulo, Brazil
}

\begin{abstract}
Aim: To evaluate cyclin-dependent kinase inhibitor $2 A(C D K N 2 A)$ and cytokeratin 7 (CK7) expression in cervical intraepithelial neoplasia (CIN) formalin-fixed samples. Materials and Methods: Staining with antibody clones G175-405 for CDKN2A and OV-TL 12/30 for CK7 were evaluated and the detection of protein expressions were compared in 147 patients with CIN. Results: Clinical follow-up of patients with CIN1 and CIN2 showed that most patients had a favorable outcome. Single $C D K N 2 A$ or $C K 7$ expression and their combined expression had a greater sensitivity and negative predictive value in CIN1, corresponding to the non-development of the disease. The positive predictive value of $C D K N 2 A$ was greater than that of $C K 7$. Combined expression of $C D K N 2 A$ and $C K 7$ showed that the sensitivity, specificity, positive predictive values, and negative predictive values had their maximum index in the CIN1 group. Analysis of combined expression of CDKN2A and CK7 showed that $85.7 \%$ of patients presented unfavorable clinical outcomes, with positive expression for both markers identified in CIN2. Conclusion: Combined expression of CK7 and CDKN2A was associated with a better diagnosis of CIN, and negative expression in CIN1/2 groups had a greater negative predictive value for patient clinical outcome.
\end{abstract}

Persistent cervical infection caused by high-risk human papillomavirus (HPV) is necessary for the development of precursor lesions and cervical cancer (1). Although carcinogenesis does not progress in the majority of infected

Correspondence to: Thais Heinke, Department of Pathology, Federal University of São Paulo - 740 Botucatu Street, Vila Clementino, São Paulo 04023-062, Brazil. Tel: +55 1155764996, Fax: +55 1155719295, e-mail: thaish1978@gmail.com

Key Words: CDKN2A, p16 expression, CK7 expression, CIN immunohistochemistry, cervical cancer. women, cervical cancer is still the second leading cause of cancer-related deaths worldwide (2, 3). Cervical intraepithelial neoplasia (CIN), and squamous intraepithelial lesion (SIL) correspond to different stages of epithelial proliferation of undifferentiated basal cells. This progresses from CIN1 [lowgrade (L)SIL], which is very similar to normal fullydifferentiated squamous epithelium, to high-grade CIN (CIN2/3) or high-grade $(\mathrm{H}) \mathrm{SIL}$, containing mostly undifferentiated cells, and then to cervical carcinoma (4-6). CIN1 (LSIL) and CIN3 (HSIL) are more easily classified, justifying conservative management for the former and ablation for the latter (6). However, the intermediate point of distinction for CIN2, between CIN1 and CIN3, is not always clear-cut; there is a significant, well-known interobserver variability in this diagnosis. Thus, the Lower Anogenital Squamous Terminology (LAST) Project recommends the use of p16 immunohistochemistry as an adjunct to the morphological assessment of biopsies when the differential diagnosis includes high-grade lesions $(7,8)$.

Cyclin-dependent kinase $2 \mathrm{~A}(\mathrm{CDKN} 2 \mathrm{~A})$ protein, also known as p16 ${ }^{\mathrm{INK} 4 \mathrm{~A}}$, is a CDK inhibitor that blocks CDK4- and CDK6mediated phosphorylation of RB transcriptional corepressor 1 (RB) to inhibit E2F-dependent transcription and cell-cycle progression (9). It is a tumor-suppressor located at chromosome 9p21 (10), and it has been shown to be a surrogate marker of high-risk (HR) HPV oncogenic activity (11). Immunostaining of CDKN2A has an important value in the diagnostic process because it is detected in almost all cases of CIN2/3, whereas reactive mimickers, such as immature metaplasia or atrophy, are negative or only show focal staining $(9,11)$. On the other hand, the results of CDKN2A staining in CIN1 vary greatly, with some lesions being completely negative or showing focal staining, whereas others show a diffuse, basal, and positive reaction (11). There are still some controversies regarding the predictive value of CDKN2A. Therefore, some studies seek to find new biomarkers to improve the detection of lesions which are at high-risk for progression. 
Another potential biomarker for HR-HPV-associated lesions is cytokeratin 7 (CK7) protein, which is synthesized by the KRT7 gene located at chromosome $12 \mathrm{q} 13.13$. It is highly expressed in the squamocolumnar junction (SCJ) of the cervix (8). SCJ cells are characterized as residual embryonic cells, vulnerable to neoplastic transformation. Thereby, some evidence suggests that the cell population at SCJ is a source of HR-HPV infection and the initiation site of cervical carcinogenesis. Intraepithelial lesions and invasive carcinomas also express CK7 in most cases. Positive expression of CK7 in CIN1 is related to a high-risk of ultimate CIN3 diagnosis compared to CK7-negative $\mathrm{CIN} 1$, suggesting that $\mathrm{CK} 7$ has a potential role as a biomarker for the identification of higher risk CIN1 (12).

Interestingly, there is a complex relationship between keratin expression of SCJ cells and CIN. CK7 is described as a marker for the progenitor cell population at the SCJ and is expressed in most CIN2/3 lesions. Currently, it is recommended that all cervical lesions with CDKN2A and CK7 positivity should be managed as borderline HSIL or a questionable SIL, even when the lesion is morphologically classed as CIN1 (5).

Therefore, the expression of CDKN2A and CK7 in precancerous lesions might be essential in order to modulate or restrict lesion progression and cancer development. Attempting to find diagnostic and prognostic biological biomarkers for these intraepithelial lesions, we performed an immunohistochemical study evaluating the expression profile of CDKN2A and CK7 in cervical epithelial cells of different degrees of squamous intraepithelial neoplasia (CIN) and in non-neoplastic cervical tissue.

\section{Materials and Methods}

Patients. This retrospective cross-sectional study was approved through the Research Ethics Committee of the Federal University of Sao Paulo - Paulista Medical School (UNIFESP/EPM) under the protocol number $892 / 2017$, and all patients previously agreed with and signed an informed consent form.

Selected samples included 147 cervical biopsies (100 with squamous intraepithelial neoplasia and 47 without neoplasia) which were collected at the Gynecological Disease Prevention Nucleus. Patients were diagnosed by colposcopical and previous histopathological studies, and the absence of neoplasia was confirmed through the cytological and histopathological examination (13). Women with any kind of immunosuppression, diabetes, organ transplantation, or pregnancy were excluded from the study. Cervical samples were distributed into CIN1, CIN2, CIN3, and control (non-neoplastic) groups, according to the classification of Richart and Barron (14).

Clinical method. Samples of ectocervix and endocervix were collected from each patient and forwarded to the Cytology Laboratory of the Gynecology Department and Pathology Laboratory of the Federal University of São Paulo. Patient reports were based on the Bethesda system (2001). Slides were analyzed independently by two pathologists (double-blinding).

Clinical data were collected from patient charts and carefully analyzed. All patients were submitted to anamneses, general
Table I. Cytokeratin 7 (CK7) expression pattern for immunohistochemistry.

\begin{tabular}{ll}
\hline Group & CK7 expression \\
\hline 1 & Negative 'pure' \\
2 & Negative with weak focal positivity in koilocytes \\
3 & Negative with moderate to strong focal positivity in koilocytes \\
4 & Homogeneously positive with moderate to strong intensity \\
5 & Homogeneously positive with weak intensity \\
6 & Heterogeneously positive with moderate to strong intensity \\
7 & Heterogeneously positive with weak intensity \\
\hline
\end{tabular}

physical, gynecological, and specular examinations for the collection of cervico-vaginal cytology and colposcopy, with a biopsy of any abnormal findings.

Conventional histopathological method. Routinely, we prepared the formalin-fixed paraffin-embedded tissue blocks (13), which were cut into 4- $\mu$ m-thick sections using an AO American optical 820 Rotary microtome (AO Instrument Company, NY, USA). After assembly onto a glass slide, the tissue was deparaffinized in xylene, rehydrated in graded alcohols, and submitted to hematoxylin and eosin (HE) staining followed by sealing with Entellan ${ }^{\circledR}$ (Merck Millipore, Darmstadt, Germany). HE-stained slides of all cases were reviewed and the diagnoses confirmed.

Slides were analyzed based on the methodology of Richart and Barron (14) and all cases were reviewed independently by two different experienced pathologists; discordant diagnoses were resolved by consensus.

Construction of tissue microarray (TMA). TMA construction was performed according to the standard technique previously described elsewhere $(13,15,16)$, using corresponding HE-stained slides as a guide. Cylinders of $0.8 \mathrm{~mm}$ diameter were punched from selected areas of each donor paraffin block, and these were mounted into a receptor paraffin block at $1 \mathrm{~mm}$ intervals using a precision microarray instrument (Beecher Instruments, Silver Spring, MD, USA). Blocks were sealed at $60^{\circ} \mathrm{C}$ for $10 \mathrm{~min}$ before the cutting of $4-\mu \mathrm{m}$ sections and preparation using standard methods. Sections were fixed onto silanized slides, and three glass slides were submitted to HE staining for the evaluation of the samples before immunohistochemistry, as previously standardized by our group (13).

Immunostaining for $C D K N 2 A$ and $C K 7$. Reactions were carried out in the Immunopathology Laboratory and Special Techniques of the Pathology Department of Federal University of São Paulo, following an automated standardized method through the immunoperoxidase activity. Specimens were incubated with Pierce $^{\mathrm{TM}}$ 3-aminopropyltriethoxysilane (Thermo Fisher Scientific, MA, USA) in an oven at $60^{\circ} \mathrm{C}$ for 24 hours. Briefly, CDKN2A and CK7 immunohistochemistry were conducted in an Autostainer Link 48 (Agilent, Dako, Santa Clara, CA, USA). Sections were dewaxed, rehydrated and treated for quenching of endogenous peroxidase activity. To enhance the antigen retrieval, PT-link buffer (Dako, Glostrup, Denmark) with pH 9.0 was used at $97^{\circ} \mathrm{C}$ for $2 \mathrm{~h}$. Sections were washed with phosphate-buffered saline $10 \mathrm{mM}$ with $\mathrm{pH} 7.4$ for $5 \mathrm{~min}$, followed by incubation with specific antibody to CDKN2A and CK7. 
Table II. Age distribution and patient outcome according to the diagnosis.

\begin{tabular}{|c|c|c|c|c|c|c|c|}
\hline & & Control & CIN1 & CIN2 & CIN3 & Total & $p$-Value \\
\hline \multirow[t]{2}{*}{ Age (years) } & Mean \pm SD & $33.7 \pm 9.9$ & $32.5 \pm 8.5$ & $32.1 \pm 8.8$ & $33.1 \pm 7.8$ & $32.9 \pm 8.8$ & $0.828^{\mathrm{a}}$ \\
\hline & Median (range) & $32.0(18-54)$ & $32.0(19-52)$ & $29.0(22-58)$ & $31.0(25-54)$ & $32.0(18-58)$ & \\
\hline \multirow[t]{4}{*}{ Outcome, n (\%) } & Unfavorable & - & $2(5.7)$ & 7 (21.9) & - & $9(6.1)$ & $0.075^{\mathrm{b}}$ \\
\hline & Favorable & - & $33(94.3)$ & $25(78.1)$ & - & $58(39.5)$ & \\
\hline & Without follow-up & $47(100)$ & - & - & $33(100)$ & $80(54.4)$ & \\
\hline & Total & $47(100)$ & $35(100)$ & $32(100)$ & $33(100)$ & $147(100)$ & \\
\hline
\end{tabular}

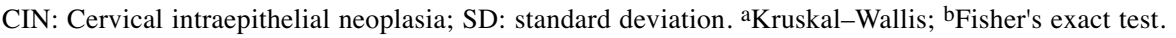

All sections were incubated with a mouse monoclonal antibody clone G175-405 to CDKN2A (Zeta Corporation, Arcadia, CA, USA) at a dilution of 1:50, followed by immersion in wash buffer for $5 \mathrm{~min}$. and amplification with polymer EnVisionFlexTM (Dako) for 20 minutes.

Sections were incubated with a FLEX monoclonal mouse antibody clone OV-TL 12/30 to CK7 (Dako), ready for use (prediluted) for $20 \mathrm{~min}$ followed by immersion in a wash buffer for 5 min and amplification with polymer EnVisionFlexTM (Dako) for 20 minutes.

After that, cervical tissues incubated with the two antibodies were washed with buffer for $5 \mathrm{~min}$ and amplified with the Novolink Novocastra Kit (Leica Biosystems, Nußloch, GE, USA). Sections were washed with buffer, which contains the chromogen 3,3'-diaminobenzidine-tetrahydrochloride-dihydrate (Sigma-Aldrich Chemical, St. Louis, MO, USA), $600 \mu$ hydrogen peroxide (V30), 100 $\mathrm{ml}$ of phosphate-buffered saline, and $1 \mathrm{ml}$ dimethyl sulfoxide for $5 \mathrm{~min}$ at $37^{\circ} \mathrm{C}$. Finally, the sections were counterstained with Harris hematoxylin and were mounted with Entellan ${ }^{\circledR}$ (Merck Millipore) (13).

Plasmocytes present on the slides were used as the internal control and squamous cell carcinoma of the uterine cervix as the external control for CDKN2A reactions. Pancreatic tissue was used as a positive external control and columnar epithelium of the uterine cervix itself as an internal positive control for CK7 reactions. Two pathologists carried out the evaluation independently. The CDKN2A reading was carried out using the LAST Consensus 2012 recommendation, i.e. continuous strong nuclear or nuclear plus cytoplasmic 'block-type' staining involving at least the basal third of the squamous epithelium was considered positive (17). CK7 expression was classified according to the intensity of immunostaining in the cytoplasm (Table I).

For statistical analysis, the samples were divided according to the expression patterns given in Table I into negative (expression patterns 1,2, and 3) and positive (expression patterns 4, 5, 6, and 7) groups.

In the clinical data interpretation, those with histopathological diagnosis of CIN3 and in situ adenocarcinoma or invasive carcinoma after 6 months were considered as having an unfavorable clinical outcome.

Statistical analysis. Analyses were performed using Excel 2010 for Windows and the R statistical package 3.3.2 version (R Core Team, Rio de Janeiro, Brazil). For a comparison of quantitative variables among the groups, the Kruskal-Wallis test was used. For a comparison of the clinical outcome or qualitative variables chisquare tests or the exact Fisher tests were performed (18). Statistical significance was established as $p<0.05$.

\section{Results}

Patients with ages ranging from 18 to 59 years old were recruited for the study. Forty-seven women aged 33.7 \pm 9.9 (mean \pm standard deviation) years comprised the control group. In the CIN1 group, there were 35 women aged $32.5 \pm 8.5$ years. In the CIN2 group, there were 32 women aged $32.1 \pm 8.8$. In the CIN3 group, there were 33 women with a mean age of $33.1 \pm 7.8$ years (Table II).

A clinical follow-up of the CIN1 and CIN2 groups showed that the majority had a favorable outcome. A minority of patients in the CIN1 and CIN2 groups showed an unfavorable outcome, with a lower trend in CIN1 compared to CIN2 (Table II).

Our findings pointed out a great number of samples with positive CK7 expression in CIN2 and CIN3 groups compared to CIN1 (Figure 1). We found a sequentially greater number of patients with positive CDKN2A expression in CIN3, CIN2, and CIN1, taking positive CDKN2A expression as when at least the basal third of the epithelium was stained (Figure 2). Combined analysis of CDKN2A and CK7 showed strong immunoexpression in the CIN3 group (Table III).

In the CIN1 group, single immunoexpression of CDKN2A or CK7 had greater sensitivity and negative predictive value (NPV), corresponding to the non-development of the disease, as well as a relevant specificity estimate. Patients with negative expression of CDKN2A and CK7 did not show unfavorable outcomes. The positive predictive value (PPV) of CDKN2A was greater than that of CK7. Both CDKN2A and CK7 biomarkers presented maximum sensitivity for unfavorable clinical outcome. The two exceptional cases with an unfavorable outcome were positive for both CDKN2A and CK7 (Table IV).

The expression profile of CDKN2A and CK7 combined showed that the sensitivity, specificity, and PPV and NPV had their maximum values in the CIN1 group. In addition, combined positive expression of these two markers identified the two patients in the CIN1 group with an unfavorable outcome (Table IV). 

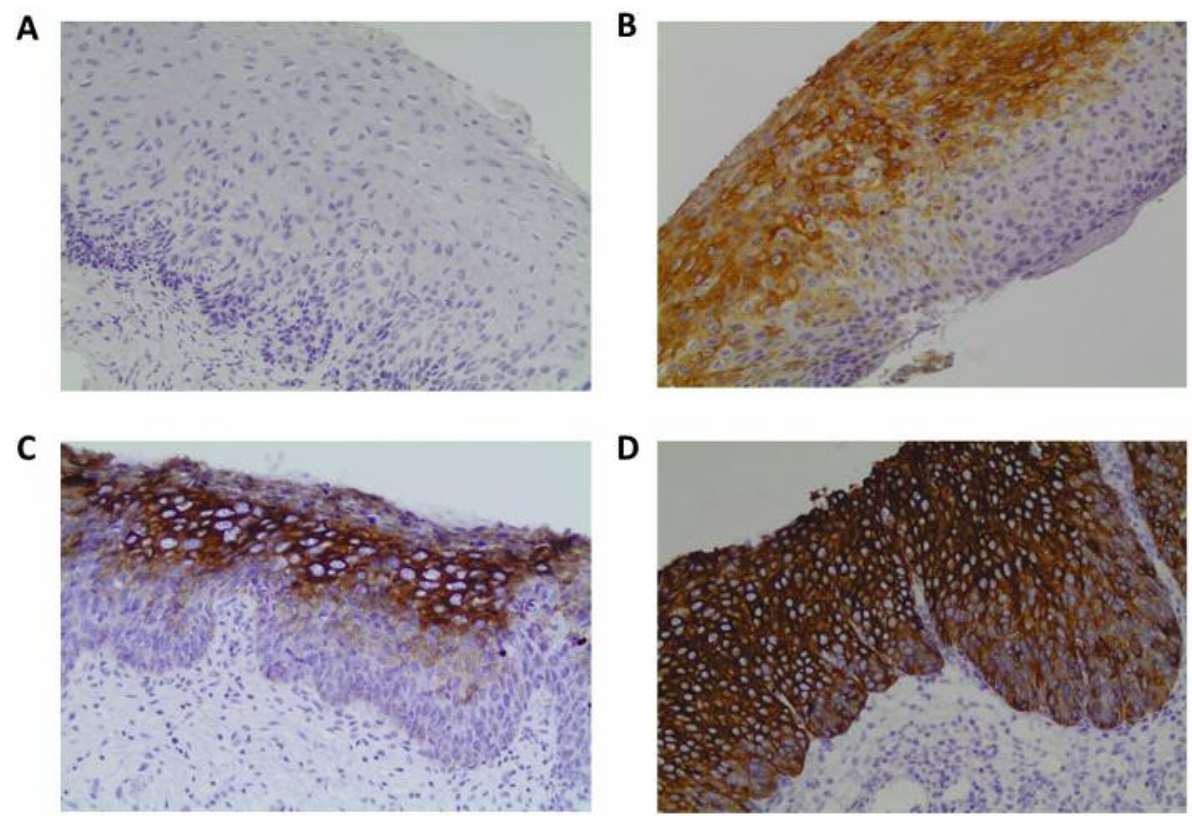

Figure 1. Microphotographs showing cytokeratin 7 (CK7) immunoexpression profile in cervical lesions. Cervical intraepithelial neoplasia (CIN) 1 with negative expression (A), CIN1 with positive expression (B), CIN2 with the superficial half of the epithelium exhibiting positive expression (C), and CIN3 with full-thickness positive expression positivity in (D) (original magnification, $\times 200$ ).

A

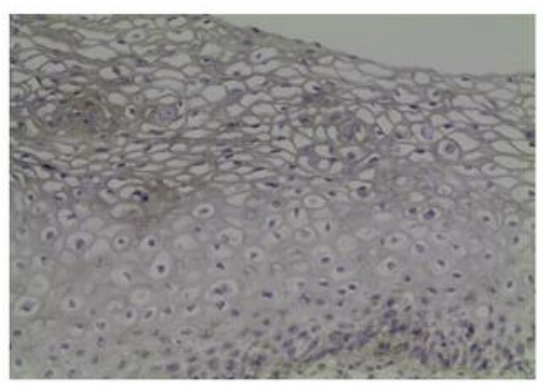

C

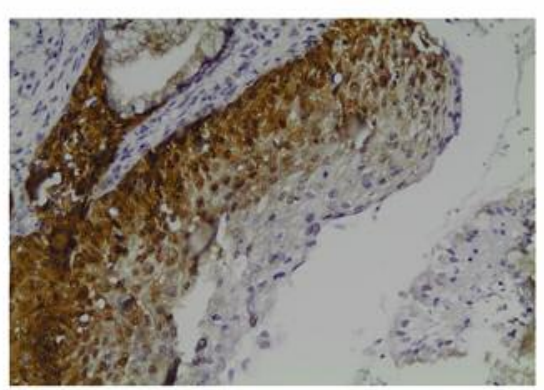

B

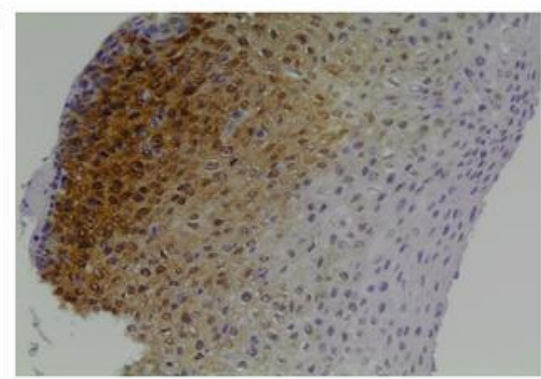

D

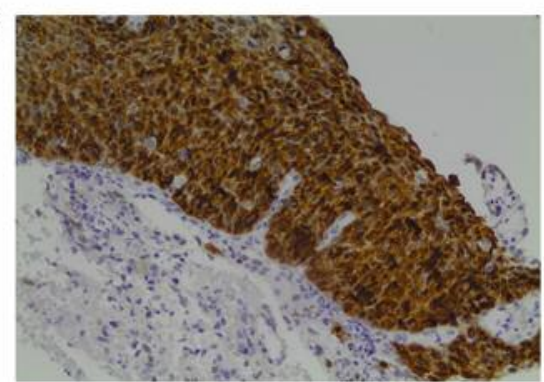

Figure 2. Microphotographs showing cyclin dependent kinase inhibitor $2 A(C D K N 2 A)$ immunoexpression profile in cervical lesions. Cervical intraepithelial neoplasia (CIN)1 with negative expression (A), CIN1 with positive expression (B), CIN2 with positive expression, and CIN3 with full-thickness positive expression (D) (original magnification $\times 200$ ).

We observed that CK7 had the maximum sensitivity and NPV for an unfavorable outcome; these values were higher than those for CDKN2A. CK7 expression identified all the patients with an unfavorable clinical outcome. Therefore, we increased the positivity threshold for CDKN2A expression that led to a specificity increase and reduced sensitivity, considered positive if the middle third was completely positive or if the immunostaining is reaching the superficial 


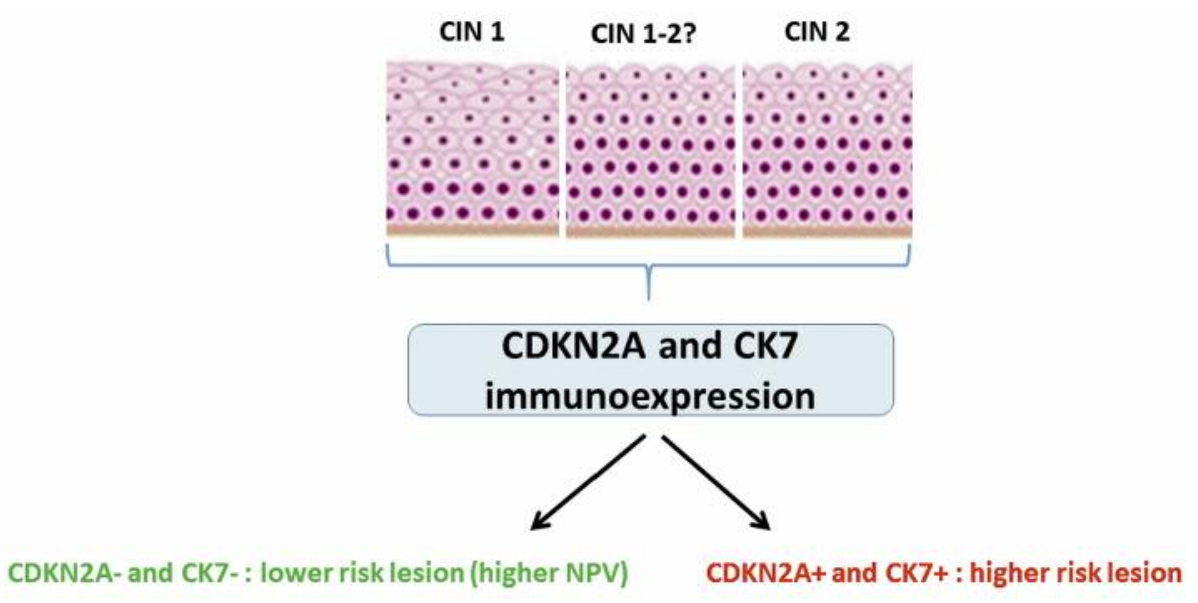

Figure 3. Potential usefulness of biomarker expression in cervical intraepithelial neoplasia (CIN)1, CIN2 and CIN of indeterminate grade (either CIN1 or 2) in order to evaluate the risk of association with cyclin-dependent kinase inhibitor $2 A$ (CDKN2A) and cytokeratin 7 (CK7).

Table III. Immunoexpression of cytokeratin 7 (CK7) and cyclin-dependent kinase inhibitor 2A (CDKN2A) in patient samples.

\begin{tabular}{|c|c|c|c|c|c|c|c|}
\hline & Immunoexpression & $\begin{array}{c}\text { Control } \\
\mathrm{n}(\%)\end{array}$ & $\begin{array}{l}\text { CIN1 } \\
\text { n (\%) }\end{array}$ & $\begin{array}{l}\text { CIN2 } \\
\mathrm{n}(\%)\end{array}$ & $\begin{array}{l}\text { CIN3 } \\
\text { n }(\%)\end{array}$ & $\begin{array}{l}\text { Total } \\
\mathrm{n}(\%)\end{array}$ & $p$-Value \\
\hline \multirow[t]{2}{*}{ CK7 } & Positive & - & $7(20)$ & $28(87.5)$ & $32(97)$ & $67(45.6)$ & $<0.001 * *$ \\
\hline & Negative & $47(100)$ & $28(80)$ & $4(12.5)$ & $1(3)$ & $80(54.4)$ & \\
\hline \multirow[t]{2}{*}{ CDKN2A } & Positive $^{\mathrm{a}}$ & - & $5(14.3)$ & $21(65.6)$ & $33(100)$ & $59(40.1)$ & $<0.001 * *$ \\
\hline & Negative & $47(100)$ & $30(85.7)$ & $11(34.4)$ & - & $88(59.9)$ & \\
\hline \multirow[t]{5}{*}{ CDKN2A/CK7 } & Positive/positive & - & $2(5.7)$ & $19(59.4)$ & $32(97)$ & $53(36.1)$ & $<0.001 *$ \\
\hline & Positive/negative & - & $3(8.6)$ & $2(6.3)$ & $1(3)$ & $6(4.1)$ & \\
\hline & Negative/positive & - & $5(14.3)$ & $9(28.1)$ & - & $14(9.5)$ & \\
\hline & Negative/negative & $47(100)$ & $25(71.4)$ & $2(6.3)$ & - & $74(50.3)$ & \\
\hline & Total & $47(100)$ & $35(100)$ & $32(100)$ & $33(100)$ & $147(100)$ & \\
\hline
\end{tabular}

CIN: Cervical intraepithelial neoplasia. *Fisher's exact test; **Chi-square test. ${ }^{a}$ Continuous strong nuclear or nuclear plus cytoplasmic 'block-type' staining involving at least the basal third of the squamous epithelium (LAST Consensus).

third. By this method, only one patient had an unfavorable clinical outcome (Table V).

Combined expression of CDKN2A and CK7 showed that $85.7 \%$ of patients presented an unfavorable clinical outcome with positive expression for both markers in the CIN2 group. The maximum NPV was associated with negative CDKN2A and CK7 expression in patients with CIN2. Sensitivity and NPV remain unchanged, even using increased criteria for CDKN2A positivity.

Figure 3 is a schematic illustration of CDKN2A and CK7 expression as potential biomarkers for intraepithelial lesions.

\section{Discussion}

The transformation zone of the uterine cervix and SCJ are the sites in which most CINs and invasive lesions caused by HPV occur. This study found variations in the expression pattern of CK7 according to type of epithelium and also the degree of CIN. Positive expression was variable in terms of extent, intensity, and topography in the different nonneoplastic and neoplastic squamous epitheliaI lesions. Due to their wide variety, we classified expression patterns more comprehensively as homogeneous or heterogeneous; the intensity was classified as weak or moderate to strong, and the extent was classified as focal or diffuse. Herfs et al. classified CK7 imunoexpression as positive when there was intense and diffuse cytoplasmic immunoreaction in more than $90 \%$ of cells in the suprabasal and apical layers $(6,19)$. However, Van der Marel et al. considered samples that stained more than $25 \%$ of cells in the basal and superficial epithelial compartments as having positive expression for CK7 protein (5), similarly to our study. The intensity of cell staining is also an important criterion for classifying CK7 expression as positive. 
Table IV. Immunoexpression of cytokeratin 7 (CK7) and cyclin-dependent kinase inhibitor $2 A(C D K N 2 A)$ in patients with cervical intraepithelial neoplasia 1 .

\begin{tabular}{|c|c|c|c|c|c|c|c|}
\hline & \multicolumn{2}{|c|}{ Outcome } & \multirow[b]{2}{*}{$p$-Value* } & \multirow[b]{2}{*}{$\begin{array}{l}\text { Sensitivity } \\
(95 \% \text { CI) }\end{array}$} & \multirow[b]{2}{*}{$\begin{array}{c}\text { Specificity } \\
(95 \% \text { CI })\end{array}$} & \multirow[b]{2}{*}{$\begin{array}{c}\text { PPV } \\
(95 \% \mathrm{CI})\end{array}$} & \multirow[b]{2}{*}{$\begin{array}{c}\text { NPV } \\
(95 \% \mathrm{CI})\end{array}$} \\
\hline & $\begin{array}{c}\text { Unfavorable, } \\
\text { n (\%) }\end{array}$ & $\begin{array}{c}\text { Favorable, } \\
\mathrm{n}(\%)\end{array}$ & & & & & \\
\hline \multicolumn{8}{|l|}{ CK7 } \\
\hline Positive & $2(100)$ & $5(15.2)$ & 0.035 & $1.000(0.342-1.000)$ & $0.849(0.691-0.933)$ & $0.286(0.083-0.641)$ & $1.000(0.879-1.000)$ \\
\hline Negative & - & $28(84.8)$ & & & & & \\
\hline Total & $2(100)$ & $33(100)$ & & & & & \\
\hline \multicolumn{8}{|l|}{ CDKN2A } \\
\hline Positive $^{a}$ & $2(100)$ & $3(9.1)$ & 0.017 & $1.000(0.342-1.000)$ & 0.909 (0.764-0.969) & $0.400(0.118-0.769)$ & $1.000(0.886-1.000)$ \\
\hline Negative & - & $30(90.9)$ & & & & & \\
\hline Total & $2(100)$ & $33(100)$ & & & & & \\
\hline \multicolumn{8}{|c|}{ CDKN2A and CK7 } \\
\hline Both positive & $2(100)$ & - & 0.003 & $1.000(0.342 ; 1.000)$ & $1.000(0.867-1.000)$ & $1.000(0.342-1.000)$ & $1.000(0.867-1.000)$ \\
\hline Both negative & - & $25(75.8)$ & & & & & \\
\hline Total & $2(100)$ & $25(100)$ & & & & & \\
\hline
\end{tabular}

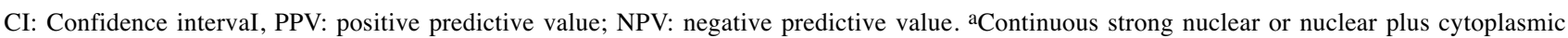
'block-type' staining involving at least the basal third of the squamous epithelium (LAST Consensus). *Fisher's exact test.

Table V. Immunoexpression of cytokeratin 7 (CK7) and cyclin-dependent kinase inhibitor $2 A$ (CDKN2A) in patients with cervical intraepithelial neoplasia 2.

\begin{tabular}{|c|c|c|c|c|c|c|c|}
\hline \multirow[b]{2}{*}{ Marker } & \multicolumn{2}{|c|}{ Outcome } & \multirow[b]{2}{*}{$p$-Value* } & \multirow[b]{2}{*}{$\begin{array}{l}\text { Sensitivity } \\
(95 \% \mathrm{CI})\end{array}$} & \multirow[b]{2}{*}{$\begin{array}{l}\text { Specificity } \\
(95 \% \mathrm{CI})\end{array}$} & \multirow[b]{2}{*}{$\begin{array}{c}\text { PPV } \\
(95 \% \mathrm{CI})\end{array}$} & \multirow[b]{2}{*}{$\begin{array}{c}\text { NPV } \\
(95 \% \mathrm{CI})\end{array}$} \\
\hline & $\begin{array}{c}\text { Unfavorable, } \\
\mathrm{n}(\%)\end{array}$ & $\begin{array}{c}\text { Favorable, } \\
\text { n }(\%)\end{array}$ & & & & & \\
\hline \multicolumn{8}{|l|}{ CK7 } \\
\hline Positive & $7(100)$ & $21(84)$ & 0.552 & $1.000(0.646-1.000)$ & $0.160(0.064-0.347)$ & $0.250(0.127-0.434)$ & $1.000(0.510-1.000)$ \\
\hline Negative & - & $4(16)$ & & & & & \\
\hline Total & $7(100)$ & $25(100)$ & & & & & \\
\hline \multicolumn{8}{|l|}{ CDKN2A } \\
\hline Positive ${ }^{a}$ & $6(85.7)$ & $15(60)$ & 0.374 & $0.857(0.487-0.974)$ & $0.400(0.234-0.593)$ & $0.286(0.138-0.500)$ & $0.909(0.623-0.984)$ \\
\hline Negative & $1(14.3)$ & $10(40)$ & & & & & \\
\hline Total & $7(100)$ & $25(100)$ & & & & & \\
\hline \multicolumn{8}{|c|}{ CDKN2A and CK7 } \\
\hline Both positive & $6(85.7)$ & $13(52)$ & $>0.999$ & $1.000(0.610-1.000)$ & $0.133(0.037-0.379)$ & $0.316(0.154-0.540)$ & $1.000(0.342-1.000)$ \\
\hline Both negative & - & $2(8)$ & & & & & \\
\hline
\end{tabular}

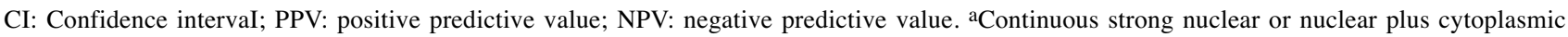
'block-type' staining involving at least the basal third of the squamous epithelium (LAST Consensus). *Fisher's exact test.

Our data showed that CK7 expression was negative in the non-metaplastic original glycogenated squamous epithelium. Similar findings were found in two different studies, which also observed that the mature metaplastic squamous epithelium presents staining only in some cells and that immature metaplastic squamous epithelium may stain more extensively and strongly for CK7 $(19,20)$. Besides this, we observed that CK7 has a tendency to stain the most superficial layers of the epithelium, corroborating Smedts $e t$ al.'s (20) findings, which showed positive expression of CK7 in the most superficial strata of the squamous epithelium. CK7 expression was localized mainly in the suprabasal layers in several studies $(6,8,21,22)$.

Interestingly, we found that immunostaining with a heterogeneous pattern was identified in a great number of samples, but the minority of CIN presented homogeneous staining for CK7. The intensity of expression also varied from weak or moderate to strong and there was statistical 
significance in the comparisons among the three groups (CIN1, CIN2, and CIN3), showing a progressive percentage increase of CK7 expression in CIN1, CIN2, and CIN3, which corroborates the data in literature $(6,19-21)$.

In the current study, $87.5 \%$ of CIN 2 and $97 \%$ of CIN3 were CK7 positive. Positive expression of CK7 has already been reported in more than a half of CIN3 cases (20), whereas Herfs et al. (6) and Paquette et al. (21) found CK7 positivity in almost all cases of CIN3 (91.8\% and 81\%, respectively). Two other studies presented positive expression in all high-risk lesions, indicating that high-grade lesions have a greater expression of CK7 $(5,19)$. In addition, a recent evaluation of 116 cervical biopsies with CIN2 histological diagnosis showed that $90.5 \%$ of cases were positive for CK7 (23). In CIN2, we observed that all patients with unfavorable clinical outcome were CK7-positive, with a sensitivity of $100 \%$ for the marker in identifying an unfavorable outcome. Umphress et al. obtained similar results (23). A few studies have evaluated the prognosis of patients with CIN2 using CK7, but no one presented the same criteria, making this work unheard of.

For CIN1, 80\% had a negative expression. In this context, Huang et al. found that $81.7 \%$ of CIN1 samples did not express CK7 (8), which was similar to our results. Nevertheless, Paquette et al. (21) observed that $34-59 \%$ of patients had CK7-positive expression in the CIN1 group, depending on the criteria used to analyze the samples.

In order to evaluate the prognostic value, we analyzed the expression profile of CK7 and CDKN2A according to the sensitivity and specificity for identifying unfavorable outcomes. Positive expression of CK7 showed maximum sensitivity for identifying unfavorable outcomes in CIN1, but five out of 33 samples with favorable outcome were CK7 positive. Herfs et al. (6) and Paquette et al. (21) noted that $24.2 \%$ and $32 \%$ of patients with LSIL, respectively, with positive expression for CK7 had an unfavorable outcome; they still observed that CK7 had the maximum NPV for unfavorable outcomes. We found that all cases of CIN1 with unfavorable outcomes were CK7 positive. Mills et al. (22) observed this same result, which shows that LSIL derived from the SCJ has a higher potential for progression to HSIL. CK7 is important for risk stratification because the stronger the intensity of tissue staining, the greater the chance of an unfavorable outcome. Thus, the difference between the groups indicates that LSILs with positive junctional markers form a more heterogeneous group than those with negative junctional markers.

The PPV for CK7 positivity was 0.286 in CIN1, while for CDKN2A it was 0.4. A maximum PPV (1.0) for combined CDKN2A and CK7 positivity was noted in our findings. Combined positive expression of CDKN2A and CK7 was associated with a greater PPV when compared to the individual expression for each marker (21). Moreover, we assessed the cytological results and found that HSIL, atypical squamous cells, cannot be excluded. High-grade squamous intraepithelial lesion and atypical glandular cell findings were associated with unfavorable outcomes in all samples, compared to isolated positive CDKN2A or CK7 immunoexpression.

The LAST consensus, together with the American Society for Colposcopy, Cervical Pathology, and the College of American Pathologists, recommend the use of CDKN2A immunohistochemistry, especially in atypical immature cervical squamous epithelia for the differential diagnosis between benign changes and precancerous lesions. In the CIN1/2 interface, CDKN2A is indicated for diagnosis of LSIL versus HSIL, but is not recommended for CIN3 and CIN1 (17). After that publication, several authors used CDKN2A as a biomarker in CIN1 samples to evaluate the risk of progression to more severe lesions, variably defined by cytological and histological diagnosis of CIN2 or worse $(8,11,24,25)$. In addition, the inclusion of CIN2 as an unfavorable outcome for CIN1 showed a greater PPV and lesser NPV than in our study. Unlike other studies, we only considered the histological confirmation of CIN3 or worse as a criterion of unfavorable progression or outcome.

Mills et al. observed that immunohistochemistry for CDKN2A does not significantly stratify the progression risk in patients with CIN1 (25). In patients with CIN1 who are at risk of progression to $\mathrm{CIN} 2 / 3$, a positive CDKN2A expression is not sufficient to establish more aggressive or immediate management because the isolated fragment of CIN1 biopsy is not adequate for the identification of HPV type, it is not necessarily concordant with the previous cytological result, and not exclude the co-existence of unsampled CIN2/3. Previous or concomitant high-grade cytology in CIN1 biopsies present greater PPV than immunohistochemistry for CDKN2A, which agrees with our findings, since all patients from the CIN1 and 2 groups with unfavorable outcome presented previous or concomitant cytology of HSIL or atypical squamous cells. Isolated CDKN2A immunohistochemistry does not have a sufficient PPV to change clinical management, once the cytology better stratifies the risk. Thereby, the recommendation of the LAST consensus is to use CDKN2A to identify HSIL in samples interpreted as CIN1 or less only when there is high-risk cytology.

In the CIN2 group, $28.5 \%$ of samples with positive CDKN2A expression presented a future diagnosis of CIN3 or worse, and $9.1 \%$ of samples with negative expression presented unfavorable outcomes, with a better NPV than PPV. Unfavorable outcome was used as a histological diagnostic criterion of CIN3 or worse, with NPV greater than the PPV. This demonstrates that a significant number of potentially serious cases could be underdiagnosed, taking into account the negative expression of CDKN2A in patients with CIN2 (26). These results were also observed in our study. 
Additionally, we evaluated two different criteria of positivity for CDKN2A expression, beyond those defined by the LAST consensus, seeking to support patterns related to a better accuracy of the marker in the prediction of unfavorable outcomes. Our results showed higher specificity associated with reduced sensitivity when the expression threshold for CDKN2A positivity was increased, but the best results were observed using the LAST consensus criteria (17). Considering the previously cited studies, CDKN2A is a relevant biomarker to detect patients who unlikely to have progression of an intraepithelial lesion but will not indicate an unfavorable prognosis regardless of the previous histological diagnosis of CIN1 or CIN2. Umphress et al. pointed out a high sensitivity for CDKN2A in predicting progression to CIN3 or worse (23), similarly to our study.

Of note, we analyzed the combination of CDKN2A and CK7 expression in the same CIN1 samples, and we observed that when expression for the two proteins was negative no patient progressed unfavorably and the NPV was at maximum. All patients who presented positive expression for both proteins had lesion 'progression'. In a previous study, only $20 \%$ of patients had disease progression (8), unlike our results. This can be explained by the methodology adopted in our study that considered progression only when CIN 3 or worse was diagnosed, while the other authors considered a diagnosis of CIN2 or worse as progression, reducing the number of patients with unfavorable evolution (8). Moreover, the pattern of positive expression for both markers was progressive, beginning with a frequency of $5.7 \%$ of CIN1 samples, followed by $59.4 \%$ of CIN2, and $97 \%$ of CIN3. Mills et al. also observed an increased chance of progression from CIN1 to CIN3 or worse when the two markers were positive (21).

A profile analysis of CDKN2A and CK7 markers showed that expression was positive for both in $64.7 \%$ of cases and was negative for $28.3 \%$. It has also been found that positive expression of CDKN2A and CK7 increases the chance of progression from CIN2 to CIN3 or worse (23). In addition, this investigation indicated that $\mathrm{CDKN} 2 \mathrm{~A}$ specificity is greater than $\mathrm{CK} 7$, as observed in the present study. Unfortunately, the limited number of samples in the present study did not allow correlation of the discordant CDKN2A and CK7 results.

Although the CDKN2A clone used in our study was considered of low sensitivity for the detection of HSIL (7), the NPV presented a high value when we used the LAST consensus criteria.

Immunohistochemistry is essential in the adequate management of patients with intraepithelial lesions with different biological risks. It is important to investigate new biological markers to improve the prognosis, diagnosis, and treatment of patients with cervical lesions, especially young women that are often submitted to unnecessary cold knife conization, large loop excision of the transformation zone, loop electrosurgical excision procedure or hysterectomy (13). Due to discrepancies in the literature about these biological markers in cervical lesions, we realized the importance of evaluating the expression profile of both CDKN2A and CK7, showing their significance as a prognostic biomarker.

Even though we have contemplated the findings in literature, we aimed to validate such findings in the Brazilian population.

In conclusion, the great majority of research focuses on the risk of progression of precursor lesions; however, it is extremely relevant to identify patients who do not present a risk of progression to CIN 2/3 or cervical cancer. Negative expression of CDKN2A and CK7 in samples from patients with CIN1 and CIN2 are associated with minimum risk of progression. More studies are necessary to further our understanding of the eventual role of these biomarkers in intraepithelial lesions and cervical cancer progression.

\section{Conflicts of Interest}

The Authors declare that there are no conflicts of interest in regard to this study.

\section{Acknowledgements}

This work was supported by the CAPES - Coordenação de Aperfeiçoamento de Pessoal de Nivel Superior and CNPq - Conselho Nacional de Desenvolvimento Científico e Tecnológico Grants.

\section{References}

1 Saslow D, Solomon D, Lawson HW, Killackey M, Kulasingam SL, Cain JM, Garcia FAR, Moriarty AT, Waxman AG, Wilbur DC, Wentzensen N, Downs LS, Spitzer M, Moscicki A-B, Franco EL, Stoler MH, Schiffman M, Castle PE, Myers ER, Chelmow D, Herzig A, Kim JJ, Kinney W, Herschel WL and Waldman J: American Cancer Society, American Society for Colposcopy and Cervical Pathology, and American Society for Clinical Pathology screening guidelines for the prevention and early detection of cervical cancer. J Low Genit Tract Dis 16: 175-204, 2012.

2 D'Abramo CM and Archambault J: Small molecule inhibitors of human papillomavirus protein-protein interactions. Open Virol J 5: 80-95, 2011 .

3 Schiffman M, Wentzensen N, Wacholder S, Kinney W, Gage JC and Castle PE: Human papillomavirus testing in the prevention of cervical cancer. J Natl Cancer Inst 103: 368-383, 2011.

4 Xue Y, Bellanger S, Zhang W, Lim D, Low J, Lunny D and Thierry F: HPV16 E2 is an immediate early marker of viral infection, preceding E7 expression in precursor structures of cervical carcinoma. Cancer Res 70: 5316-5325, 2010.

5 Van Der Marel J, Van Baars R, Alonso I, Del Pino M, Van De Sandt M, Lindeman J, Ter Harmsel B, Boon M, Smedts F, Ordi J, Torné A, Jenkins D and Quint W: Oncogenic human papillomavirus-infected immature metaplastic cells and cervical neoplasia. Am J Surg Pathol 38: 470-479, 2014. 
6 Herfs M, Parra-Herran C, Howitt BE, Laury AR, Nucci MR, Feldman S, Jimenez CA, McKeon FD, Xian W and Crum CP: Cervical squamocolumnar junction-specific markers define distinct, clinically relevant subsets of low-grade squamous intraepithelial lesions. Am J Surg Pathol 37: 1311-1318, 2013.

7 Shain AF, Wilbur DC, Stoler MH, Quade BJ and Kong CS: Test characteristics of specific p16 clones in the detection of highgrade squamous intraepithelial lesions (HSIL). Int J Gynecol Pathol 2: 1, 2017.

8 Huang EC, Tomic MM, Hanamornroongruang S, Meserve EE, Herfs $M$ and Crum CP: p16 INK4 and cytokeratin 7 immunostaining in predicting HSIL outcome for low-grade squamous intraepithelial lesions: a case series, literature review and commentary. Mod Pathol 29: 1501-1510, 2016.

9 Sarma U, Biswas I, Das A, Das GC, Saikia C and Sarma B: p16 ${ }^{\text {INK4A }}$ expression in cervical lesions correlates with histologic grading - a tertiary level medical facility based retrospective study. Asian Pac J Cancer Prev 18: 2643-2647, 2017.

10 Mahajan A: Practical issues in the application of p16 immunohistochemistry in diagnostic pathology. Hum Pathol 51: 64-74, 2016

11 Sagasta A, Castillo P, Saco A, Torné A, Esteve R, Marimon L, Ordi J and Del Pino M: P16 staining has limited value in predicting the outcome of histological low-grade squamous intraepithelial lesions of the cervix. Mod Pathol 29: 51-59, 2016.

12 Lee H, Lee H and Cho YK: Cytokeratin 7 and cytokeratin 19 expression in high-grade cervical intraepithelial neoplasm and squamous cell carcinoma and their possible association in cervical carcinogenesis. Diagn Pathol 12: 18, 2017.

13 Belfort-Mattos PN, Focchi GR de A, Ribalta JCL, Megale De Lima T, Nogueira Carvalho CR, Kesselring Tso F and De Góis Speck NM: Immunohistochemical expression of VEGF and podoplanin in uterine cervical squamous intraepithelial lesions. Dis Markers 2016: 1-8, 2016.

14 Richart RM and Barron BA: The intrauterine device and cervical neoplasia. A prospective study of patients with cervical dysplasia. JAMA 199: 817-819, 1967.

15 Tawfik El-Mansi M and Williams ARW: Validation of tissue microarray technology using cervical adenocarcinoma and its precursors as a model system. Int J Gynecol Cancer 16: 12251233, 2006.

16 Kononen J, Bubendorf L, Kallioniemi A, Bärlund M, Schraml P, Leighton S, Torhorst J, Mihatsch MJ, Sauter G and Kallioniemi OP: Tissue microarrays for high-throughput molecular profiling of tumor specimens. Nat Med 4: 844-847, 1998.

17 Darragh TM, Colgan TJ, Cox JT, Heller DS, Henry MR, Luff RD, McCalmont T, Nayar R, Palefsky JM, Stoler MH, Wilkinson EJ, Zaino RJ and Wilbur DC: The Lower Anogenital Squamous Terminology Standardization Project for HPV-Associated Lesions: Background and consensus recommendations from the College of American Pathologists and the American Society for Colposcopy and Cervical Pathology. J Low Genit Tract Dis 16: 205-242, 2012.
18 Agresti A: An introduction to categorical data analysis second edition. Wiley \& Sons, Inc., Hoboken, NJ, USA, 2007.

19 Herfs M, Yamamoto Y, Laury A, Wang X, Nucci MR, McLaughlin-Drubin ME, Münger K, Feldman S, McKeon FD, Xian W and Crum CP: A discrete population of squamocolumnar junction cells implicated in the pathogenesis of cervical cancer. Proc Natl Acad Sci USA 109: 10516-10521, 2012.

20 Smedts F, Ramaekers F, Link M, Vooijs GP, Lauerova L, Troyanovsky S and Schijf C: Detection of keratin subtypes in routinely processed cervical tissue: implications for tumour classification and the study of cervix cancer aetiology. Virchows Arch 425: 145-155, 1994.

21 Paquette C, Mills AM and Stoler MH: Predictive value of cytokeratin 7 immunohistochemistry in cervical low-grade squamous intraepithelial lesion as a marker for risk of progression to a high-grade lesion. Am J Surg Pathol 40: 236-243, 2016.

22 Mills AM, Paquette C, Terzic T, Castle PE and Stoler MH: CK7 immunohistochemistry as a predictor of CIN1 progression. Am J Surg Pathol 41: 143-152, 2017.

23 Umphress B, Sanchez B, Paintal A, Nayar R and Maniar KP: Utility of CK7 versus p16 as a prognostic biomarker in CIN2. Am J Surg Pathol 42: 479-484, 2018.

24 Pacchiarotti A, Ferrari F, Bellardini P, Chini F, Collina G, Dalla Palma P, Ghiringhello B, MacCallini V, Musolino F, Negri G, Pisa R, Sabatucci I and Giorgi Rossi P: Prognostic value of p16INK4A protein in women with negative or CIN1 histology result: A follow-up study. Int J Cancer 134: 897-904, 2014.

25 Mills AM, Paquette C, Castle PE and Stoler MH: Risk stratification by p16 immunostaining of CIN1 biopsies: A retrospective study of patients from the quadrivalent HPV vaccine trials. Am J Surg Pathol 39: 611-617, 2015.

26 Maniar KP, Sanchez B, Paintal A, Gursel DB and Nayar R: Role of the biomarker p16 in downgrading-CIN 2 diagnoses and predicting higher-grade lesions. Am J Surg Pathol 39: 1708$1718,2015$.
Received November 6, 2018

Revised November 16, 2018

Accepted November 19, 2018 